\title{
Research and Implementation of Key Technology of Computer System Structure Experimental Cloud
}

\author{
Feng Zhou \\ Jiangxi Technical College of Manufacturing, Department of Information \\ Engineering,Nanchang,330095
}

Keyword: Remote experiment; Cloud computing; System structure; Resource scheduling algorithm

\begin{abstract}
Remote experiment is a good solution, but the remote experiment platform is mainly provided by the virtual environment, which is the lack of real hardware accuracy and experimental experience. The development and application of cloud computing technology provides a new design concept and technical support for the remote experiment. Cloud computing is a business service model that provides infrastructure, platforms and applications as services to users. The application of cloud computing in remote experiments has brought many advantages: technology and equipment threshold reduction; convenience development and maintenance; "light client" thinking, easy access; more experimental content and functions. Under this background, this paper uses the experimental mode of "remote experiment" + "hardware device" to provide users with the hardware experimental device which is taken as a service, so that users can apply for hardware devices to conduct autonomous experiments anytime and anywhere through the network.
\end{abstract}

\section{Introduction}

Experiment is an indispensable part of computer course teaching. It is an effective way to cultivate students 'abilities and increase students' understanding of course content. With the popularity of computers and the Internet, remote learning has gradually become well known. Especially in recent ten years, with the advance of network technology and the increase of network bandwidth, remote education has gradually developed and formed a large-scale industry. At present, the remote laboratory has been widely popularized and applied in the experimental teaching of various disciplines.Under this background, this paper uses the experimental mode of "remote experiment" + "hardware device" to provide users with the hardware experimental device which is taken as a service, so that users can apply for hardware devices to conduct autonomous experiments anytime and anywhere through the network, which makes this research be more significance.

\section{Cloud Computing}

Cloud computing industry is considered to be the fourth revolution in IT industryfollowing the mainframe, personal computers and the Internet.With the three networks convergence, internet of things and information security, cloud computing has become a new engine to vigorously promote the construction of national informatization and economic development with information sharing and interconnection as the key points.

At present, there are mainly three types of cloud computing architecture: Saa S, Paa S and Iaa S. There is no uniform definition of the concept of cloud computing, but the cloud or the cloud platform should have the following properties: The cloud must be embodied as a service transaction rather than a physical transaction; cloud providers have a certain size of infrastructure (such as adequate network bandwidth, as well as servers and storage); cloud providers provide customers a renting services of the resource rather than a sale of the resource.

\section{Cloud Computing Research and Its Applications in Remote Experiments}

As the hottest topic in computer industry and academia, cloud computing technology has been widely used in many fields and the concept of cloud is everywhere. With the popularity of "cloud" 
ideas, many technologies have undergone tremendous changes, and remote experimentation is one of them. The application of cloud computing in remote experiments has brought many advantages for the construction of remote laboratories.

Technology Threshold Reduction. Remote lab construction in many disciplines (such as advanced networks and distributed systems) has been supported by a wide variety of IT technologies, in which Service-Oriented Architecture and Grid are the typical representatives.

Easy Access. of the major changes that cloud computing has brought to remote experiments is the widespread use of B / S. Since cloud computing advocates the concept of "light client", it dissects computing tasks and infrastructure from the client, and the load on the user terminal is reduced, which is mainly used to provide an experimental interface and collect user experimental information. Therefore, the browser becomes the best form to access to experimental service.

More Complete Functions. Because cloud computing systems provide a large-scale infrastructure and have developed a complete set of underlying hardware resource management and maintenance mechanisms, cloud computing technology can support more remote experiment types and experiment contents.As the cloud computing system provides hardware devices and independently manages the devices, the development and management of remote experiments are simplified and the capital investment is reduced. Therefore, the number of remote experiments in different fields is constantly increasing.

\section{Research on Key Technology of Remote Experiment}

Virtualization is the key technology in cloud computing. Through virtualization, a single server can generate multiple server virtual images to run multiple operating systems and applications, thereby improving the utilization of physical devices and providing technical support for the scalability of cloud computing platforms.

The principle behind virtualization is to create the execution environment for the program or software which it is running. The program or software is not exclusive on the underlying physical resources. What it has is only a mirror to the hardware it needs (CPU Frequency, memory and memory capacity) and this mirror may be completely different from the real hardware conditions. Therefore, the use of virtualization technology can shield the complexity of the underlying hardware devices, as shown in Fig. 1.

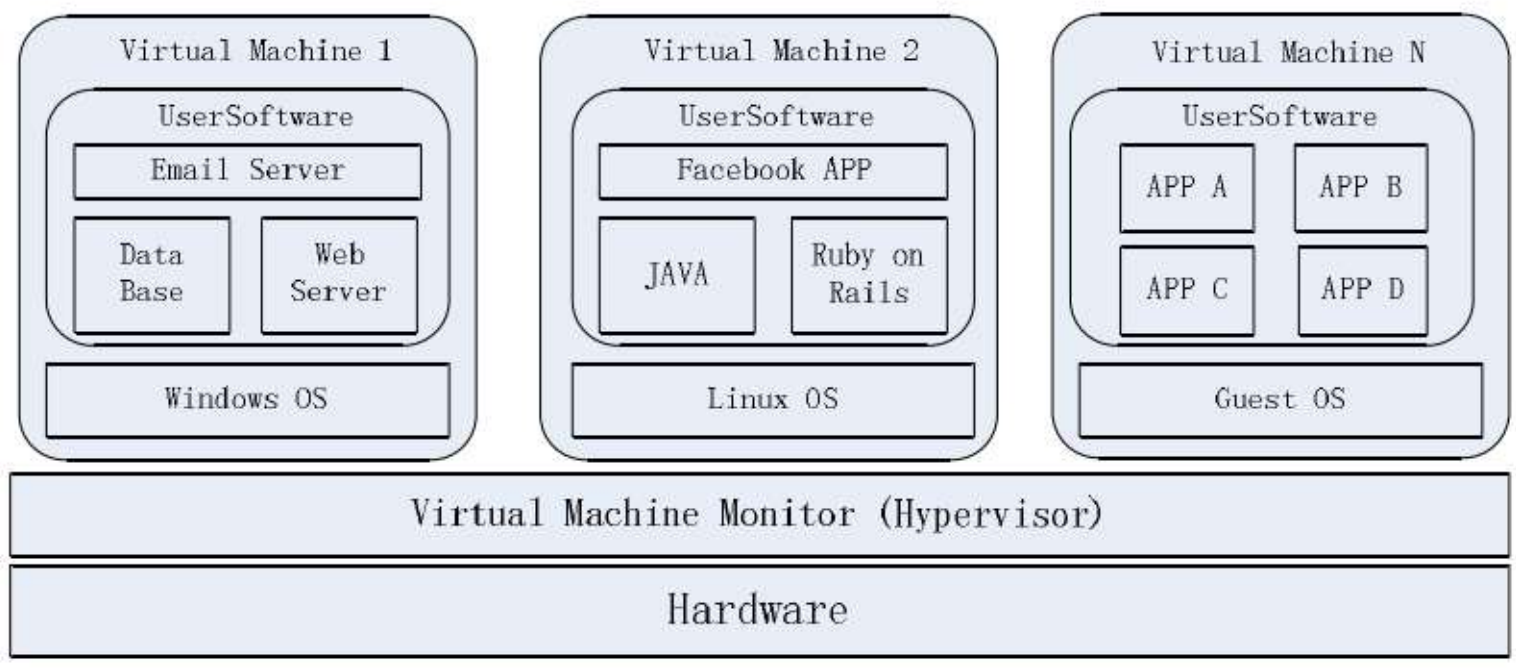

Figure 1. Application of virtualization technology

\section{Computer System Architecture Experimental Cloud System Architecture}

Experimental cloud is tries to establish a comprehensive practice teaching system which has moderate scale, resource sharing, easy use and first-class management. For this reason, the 
following functions are mainly considered in the construction of system structure experimental cloud prototype system:

Remote Experiment. experiment is one of the core functions of system structure experimental cloud. After the user logs in to the client through the browser or downloads the application, he or she can apply for an idle board, submit his or her own configuration file and program code to the server, and finally download it to the experiment board.

Download. In order to enable users to grasp the principle and method of some course experiments as soon as possible and improve the ability of users to independently carry out experiment, the system structure experimental cloud platform portal provides experimental and course material downloading areas.

Experiment Progress Preservation and Recovery. users do large-scale programming and cannot finish in one time, we provide on-site saving function and bind with user name. When the user logs in the next time, he or she can choose to resume the site to continue the experimental design.

The overall framework of the experimental platform is designed, which is shown in Fig. 2.

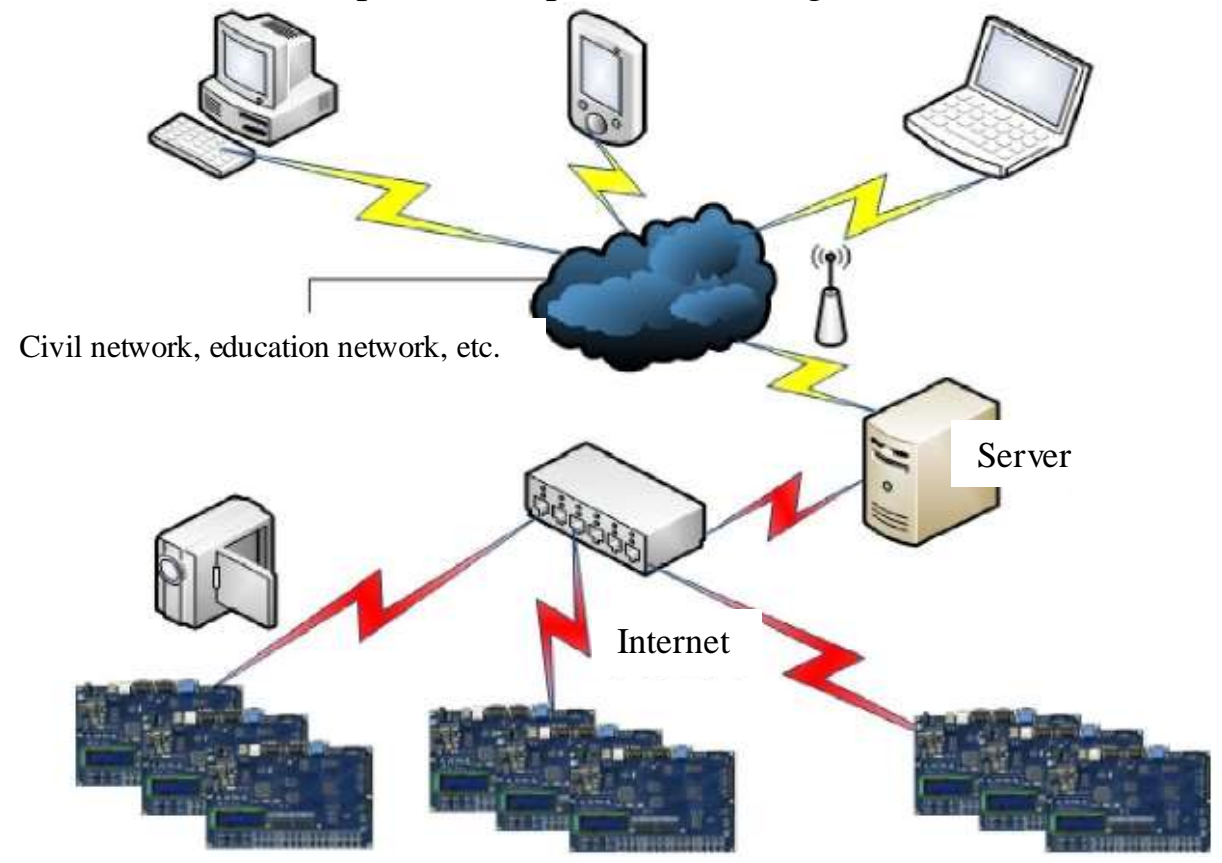

Figure 2. Overall framework of the experimental platform

It can be seen from Fig. 2, the overall structure of the remote experiment platform is divided into 4 parts, which are client, server, experiment server and the hardware connecting with the experiment server.

\section{Experimental Cloud Resource Scheduling Algorithm and Network Optimization}

One of the characteristics of system structure experimental cloud is the user's experimental program is real hardware. Due to the fact that experimental boards must be operated exclusively, each experimental board can only support one user at a time. Therefore, the management and effective scheduling of the experimental board directly determines the resource utilization rate and the number of users that can be simultaneously supported of the experimental cloud system.

The resource scheduling algorithm designed for experimental cloud system mainly considers three functions: the choice of the experimental board, the management of the experimental board and the selection of the requesting users. As shown in Fig. 3.

The three functions correspond to the following three cases. (1) When the number of users is less than that of free circuit boards, which circuit board shall be chosen for the user. (2) how to manage the experiment board so that it can be time-shared for more users at the same time. (3) which user to 
respond to when the number of users submitting experimental requests exceeds that of free experimental boards. The following describes these three situations.

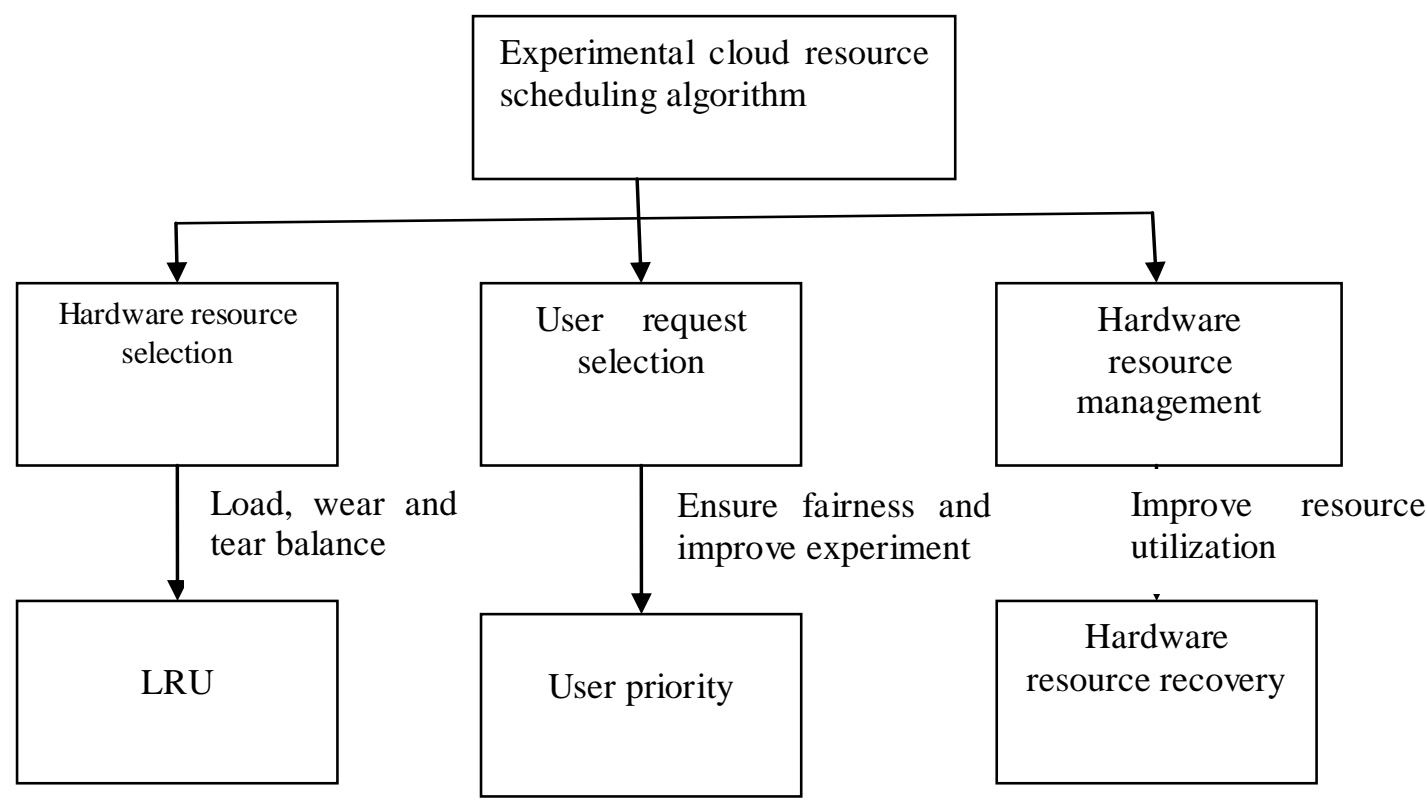

Figure 3. Experimental cloud resource scheduling algorithm framework

\section{Conclusion}

There are many shortcomings, such as waste of resources, experimental time and limited location in the traditional experimental methods. At present, the mainstream remote laboratories mostly use the virtual experimental environment, which lacks the accuracy of the real hardware and perceptual awareness.Meanwhile, the rapid development and maturity of cloud computing technology provides a new idea for the work of this paper. As a business model, cloud computing has a profound impact on the transformation of IT industry. In the cloud thinking, whether infrastructure, platform or software can be provided as a service to users. The cloud provides a resource renting service rather than a resource transaction. Users request services on demand, without concerns for the properties of the resource itself.At present, many IT vendors have launched their own cloud computing platforms, where the computing resources and storage resources are provided as services to users. In this context, this paper attempts to build a new experimental mode of "remote experiment" + "hardware", providing hardware experimental equipment to users as a service form, so that users do not need to purchase equipment and can remotely utilize the equipment to complete their own experiments.

\section{References}

[1] Tang J, Meng Y. Research and Implementation of Intelligent Traditional Chinese Headdress System in Computer Animation[J]. Applied Mechanics \& Materials, 2014, 519-520:473-476.

[2] Li H. Research and implementation of skills training platform based on cloud computer system[J]. Experimental Technology \& Management, 2015.

[3] Shen J. Research and Implementation of Computer Network Security Monitoring System[J]. China Computer \& Communication, 2017.

[4] Dai C, Zhao W, Liu J, et al. Research and implementation of navigation computer test system[J]. Electronic Measurement Technology, 2014. 
[5] Ming X. Research and system Design and Implementation of Computer Forensics Based on $\log [\mathrm{C}] / /$ The, Eai International Conference on Multimedia Technology and Enhanced Learning. 2017.

[6] Chen X, Chen Z, Li H, et al. Research and implementation of the spectrum analysis system based on FPGA[J]. Electronic Measurement Technology, 2016.

[7] Jiang L. A Corporate Computer Network Security System Design and Implementation[J]. Advanced Materials Research, 2014, 1044-1045:965-967.

[8] Yin-Chuan H E, Sun D X, Wang J, et al. Research and Implementation of Mobile Phone Vibrator Based on Android System[J]. Modern Computer, 2014.

[9] Golz J, Biesenbach R. Implementation of an autonomous chess playing industrial $\operatorname{robot}[\mathrm{C}] / /$ International Conference on Research and Education in Mechatronics. IEEE, 2016:53-56.

[10] Kortli Y, Marzougui M, Atri M. Efficient implementation of a real-time lane departure warning system[C]// Image Processing, Applications and Systems. IEEE, 2017:1-6.

[11]Wang W Q. The Application Research of Campus Card Teacher Attendance System[J]. Computer Knowledge \& Technology, 2016.

[12] Aina L V, Rui Y U, Mengyao L U, et al. The research of library service system based on Android platform[J]. Intelligent Computer \& Applications, 2017. 